which could benefit the cell. The relationship between transposable elements and their host cell is made more complex by combative strategies that affect regular cellular functions. Undoubtedly, the future holds more surprises about the conflicts and collaborations between mobile elements and their host cell that contribute to genome function.

Daniel F. Voytas is in the Arnold and Mabel Beckman Center for Transposon Research, Department of Genetics, Cell Biology and Development, University of Minnesota, Minneapolis, Minnesota 55455, USA. e-mail: voytas@umn.edu

\title{
OPTICS
}

\section{Against the spread of the light}

\author{
Kishan Dholakia
}

\section{Light that propagates without spreading or diffracting sounds like a theorist's pipedream. But it is a very real proposition, and could be used to illuminate some profound aspects of wave-particle duality.}

The law of wave diffraction is a tough nut to crack. Consider, for example, a beam from a simple laser pointer. It might still seem narrow and pencil-like when you shine it on a wall or screen a few metres from you, but if you were to point it at the Moon, it would have a diameter of several hundred kilometres. But there are ways to beat the system, and one of them is investigated by Siviloglou et al. ${ }^{1}$, writing in Physical Review Letters. They have generated objects known as Airy wave packets that are entirely resistant to spreading or diffraction.

To appreciate the context of this advance, we must go back more than 100 years, to the time when it was becoming ever more apparent that classical mechanics and classical optics had significant shortcomings. Isaac Newton had originally proposed the idea that light came in discrete packets, which he called 'corpuscles'. But the wave-like behaviour of many optical phenomena swayed scientists towards the wave description of light.

With the advent of quantum mechanics, however, came the notion of wave-particle duality, which pops up in many different guises in physics. In this context, the concept of a wave packet takes on a pivotal, unifying significance. The wave packet is an 'envelope' containing an arbitrary number of waveforms, and also describes the probability that the particle or particles in a certain state will be measured to have a particular position and momentum. Owing to dispersion - waves of different frequencies travelling at different speeds - this wave packet should not remain unaltered. More generally, a given wave packet would then spread or diffract as it moves, changing its shape. wave-like behaviour of matter.
1. Cam, H. P., Noma, K., Ebina, H., Levin, H. L. \& Grewal, S. I. S. Nature 451, 431-436 (2008).

2. Earnshaw, W. C. et al. J. Cell Biol. 104, 817-829 (1987).

3. Irelan, J. T., Gutkin, G. I. \& Clarke, L. Genetics $157,1191-1203$ (2001).

4. McClintock, B. Science 226, 792-801 (1984).

5. Kipling, D. \& Warburton, P. E. Trends Genet. 13, 141-145 (1997).

6. Lin, R. et al. Science 318, 1302-1305 (2007).

7. Feschotte, C. \& Pritham, E. J. Annu. Rev. Genet. 41, 331-368 (2007).

8. Okada, T. et al. Cell 131, 1287-1300 (2007).

9. Slotkin, R. K. \& Martienssen, R. Nature Rev. Genet. 8, 272-285 (2007)

10. Hansen, K. R. et al. Mol. Cell. Biol. 25, 590-601 (2005).

11. Folco, H. D., Pidoux, A. L., Urano, T. \& Allshire, R. C. Science 319, 94-97 (2008) by Michael Berry and Nandor Balazs almost 30 years ago ${ }^{2}$. They are named after the nineteenth-century British astronomer George Biddell Airy, whose Airy function describes mathematically the way in which a star - an 'ideal' point source of light - is seen in a telescope. Owing to light's wave nature and the telescope's limited aperture, the ideal point image becomes a series of concentric ripples of bright and dark regions. In another demonstration of the underlying connections between matter and light, the Airy function is also the solution to Schrödinger's equation for a particle confined in a triangular-shaped potential well.

Siviloglou and colleagues ${ }^{1}$ shone a beam of visible light on a device known as a spatial light modulator. This is an array of liquid-crystal droplets that can present a varying path length to the wavefront of the incident light. Its effect is to mould the wavefront to a desired shape or function. The authors programmed it to produce an Airy function (Fig. 1) and looked at the beam's propagation characteristics. They confirmed that, as had been predicted ${ }^{2}$, the intensity profile of a beam of this shape remained unchanged ('propagation invariant') as the beam moved through free space.

They also verified other intriguing aspects of these beams, such as the ability of local intensity maxima in the Airy beam to be coaxed to perform ballistic dynamics, rather like a cannonball, and to follow a parabolic trajectory. Features within the beams were seen to 'accelerate' by starting to move transverse to their direction of propagation. This behaviour may mimic that of a quantummechanical particle placed in a constant gravitational field ${ }^{3}$. It would be interesting to see if one could generate such wave packets with matter waves, perhaps in a 'quantum degenerate gas' in which most atoms have settled into a ground state, creating a coherent matter wave.

In the absence of spreading or diffraction in light or matter, exciting applications become possible. Other light modes, such as Bessel beams ${ }^{4}$, have shown similar intriguing diffraction-free properties. These beams are now used to exert forces on minuscule particles (such as biological cells) in microfluidic environments, and to achieve targeted drug delivery into cells using ultrashort bursts of non-diffracting light ${ }^{5}$, with no precise focusing required. Stopping the spread of light or matter is a theorist's dream, but one with eminently practical consequences.

Kishan Dholakia is in the School of Physics and Astronomy, University of St Andrews, North Haugh, St Andrews KY16 9SS, UK. e-mail:kd1@st-andrews.ac.uk

ematical form of the Schrödinger equation (for particles) and the equation describing the diffraction of light. Light offers a very powerful way to explore such wave packets and their evolution, and to make telling analogies to the

The non-diffracting Airy wave packets generated by Siviloglou et al. ${ }^{1}$ were predicted
1. Siviloglou, G. A. Borky, J., Dogariu, A. \& Christodoulides, D. N. Phys. Rev. Lett. 99, 213901 (2007)

2. Berry, M. V. \& Balazs, N. L. Am. J. Phys. 47, 264-267 (1979).

3. Greenberger, D. M. Am. J. Phys. 48, 256 (1980).

4. Durnin, J., Miceli, J. J. Jr \& Eberly, J. H. Phys. Rev. Lett. 58, 1499-1501 (1986).

5. Tsampoula, X. et al. Appl. Phys. Lett. 91, 053902 (2007) 